\title{
Basiswissenschaften sind wichtig
}

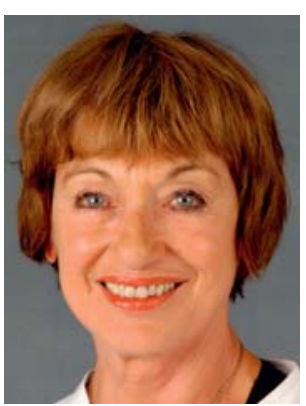

Warum haben Sie die Dermatologie als Fachgebiet gewählt?

Prof. E.-B. Bröcker: Eigentlich war es ein glücklicher Zufall. Ich war im vierten Jahr in der Immunologie in Kiel, als mein Mann 1975 einen Ruf auf eine Professur für Mathematik in Münster erhielt. Nach einem Vortrag bei einem Immunologenkongress in Basel sprachen mich Egon Macher (Chef der Münsteraner Hautklinik) und Clemens Sorg (Leiter der experimentellen Dermatologie in Münster) an und fragten, ob ich an die Hautklinik kommen wollte. Ich kam und begann im Dezember 1975 mit der Dermatologie, was ich niemals bereut habe.

Welcher Fall ist Ihnen besonders im Gedächtnis geblieben? Was war Ihr außergewöhnlichster Fall?

Prof. E.-B. Bröcker: In Münster war es Marcel, ein damals 5-jähriger Junge mit einem Hyper-IgE-Syndrom, den ich bis zu seinem 14. Lebensjahr betreut habe. Eindrucksvoll war eine temporäre „Heilung“ aller Hautprobleme, nachdem er die Masern überstanden hatte.

In Würzburg war es eine Patientin mit einem Gleich-Syndrom. Diese Diagnose - ich hatte davon noch nie gehört! - wurde von einem frisch habilitierten Oberarzt gestellt. Und dieser, es war Matthias Goebeler, wurde nach vielen Jahren - er ging als Professor nach Mannheim und wurde zunächst Chef in Gießen - mein Nachfolger in Würzburg!

Von wem haben Sie besonders viel gelernt?

Prof. E.-B. Bröcker: Oh, das waren viele Personen: Zunächst von meiner Mutter, die Hausärztin in Bielefeld war, habe ich gelernt, genau hinzusehen und zuzuhören und den Menschen dienen zu wollen. Von Prof. Müller-Ruchholtz, dem Kieler Immunologen, habe ich die Bedeutung von Kontrollen bei Experimenten gelernt. In Münster habe ich von Clemens Sorg für die Forschung etwas besonders Wichtiges gelernt, nämlich unbeirrt durch „Moden“ originell und nah an der Wirklichkeit biologischer Vorgänge zu arbeiten. Von Fereydoun Vakilzadeh habe ich - jahrelang neben ihm am Mikroskop in Münster sitzend - die Dermatohistologie erlernt, von Rudolf Happle und meiner Freundin/ Kollegin Gisela Bonsmann das Lesen dermatologischer Publika- tionen und von unserem hochverehrten Chef Egon Macher alles: Menschenliebe, Liebe zum Detail, Verbinden von Klinik mit Wissenschaft und auch, dass Dermatologie nicht das Einzige im Leben eines Dermatologen sein muss.

Nach Würzburg kam mit mir als leitender Oberarzt Henning Hamm. Von ihm habe ich wohl mehr gelernt als er von mir: Seine Kenntnisse der Genetik in unserem Fach haben mein eher immunologisch/onkologisch geprägtes Wissen in hohem Maße bereichert. Durch meine jüngeren Mitarbeiter habe ich gelernt, wie man Menschen führt, vor allem aber haben die Jungen mich auch in Frustzeiten immer wieder durch ihre Begeisterung stimuliert. Kurzum, es waren viele Menschen im Beruf, denen ich sehr viel zu verdanken habe.

Was war der beste Rat, den Sie während Ihrer Karriere erhalten haben?

Prof. E.-B. Bröcker: Zwei berühmte ältere Kollegen aus Köln und München nahmen mich 1992 als frischgebackene Klinikdirektorin beiseite: Prof. Steigleder riet mir, möglichst nah an der Hautklinik Würzburg zu wohnen (befolgt!), und Prof. Braun-Falco sagte: „Ganz gleich, ob Sie fleißig oder weniger fleißig sind. - Sie werden vier Jahre brauchen, bis Sie die Klinik im Griff haben (stimmte haargenau!).

Was ist momentan die wichtigste Entwicklung in der Dermatologie?

Prof. E.-B. Bröcker: Für mich sind es die fantastischen Fortschritte in der Dermatoonkologie - dasselbe gilt für die schon etwas länger anwendbaren Fortschritte in der Therapie entzündlicher Dermatosen.

Wo sehen Sie die Zukunft der Dermatologie?

Prof. E.-B. Bröcker: Die Antwort auf diese Frage fällt mir schwer ich bange ein wenig ob der immensen Kosten der neuen Therapien, die die Dermatologie so sehr bereichern, aber für niedergelassene Dermatologen nur schwer umzusetzen sind. Man kann hoffen, dass sich dies in naher Zukunft verbessert.

Was raten Sie jungen Kollegen?

Prof. E.-B. Bröcker: Diese Antwort fällt mir leicht: 1. Lernt Histologie, um bessere Kliniker zu werden! 2. Geht für einige Zeit in die experimentelle Forschung; auch dadurch werdet Ihr bessere Kliniker.

Was machen Sie nach Feierabend als Erstes?

Prof. E.-B. Bröcker: Im Sommer gehe ich mit einer Gartenschere bewaffnet durch den Garten, im Winter aktiviere ich meinen Mann und/oder Freunde, mit in die Oper zu kommen. 